\title{
Evaluation of Sealing Properties to LED Light Emitting Substrate of Organic-inorganic Hybrid Materials Composed of Polysilsesquioxane with Thiol Group
}

\author{
Akito HAMASUnA ${ }^{1}$, Yoshinari TAKAO ${ }^{2}$, Akihiro OTA ${ }^{2}$, Yoshihiro OHZUNO ${ }^{1}$, Takayuki TAKEI ${ }^{1}$, and Masahiro YoshIDA ${ }^{1}$ * \\ ${ }^{1}$ Graduate School of Science and Engineering, Kagoshima University, 1-21-24 Kori moto, Kagoshima city, Kagoshima 890-8580, \\ Japan
}

${ }^{2}$ YEJ Glass Corporation, 3816-23 ohkubo, Takaotyou, Izumi, Kagoshima 899-0401, Japan

\begin{abstract}
Novel organic-inorganic hybrid materials were developed as LED sealants. Polysilsesquioxane with an -SH group (PSQ-SH) and acrylic monomer were selected as the inorganic and organic components, respectively. Trimethylolpropane trimethacrylate (TRIM), which is a trifunctional monomer, was selected as an acrylic monomer, and curing of the material was performed by the thiol-ene reaction. The LED was sealed using the prepared PSQ-SH/TRIM hybrid material (PTH), and a light emission test was performed. However, cracks occurred in the hybrid material during lighting. Therefore, ditrimethylol propane tetraacrylate (DTMPTA), which is a tetrafunctional monomer, was selected for the first time to prepare a PSQ-SH/DTMPA hybrid material $(\mathrm{PDH})$ in order to enhance the strength and heat resistance of the material. The characteristics of the novel PDH were evaluated and compared with those of PTH. Specifically, the heat resistance was evaluated by confirming the onset temperature of weight reduction using thermogravimetry-differential thermal analyzer (TG-DTA) analysis, and the mechanical strength was also evaluated by bonding the glass substrates with a hybrid material and measuring the sealing strength. In conclusion, it was possible to suppress crack generation during lighting by using PDH instead of PTH in the light emission test.
\end{abstract}

\section{Introduction}

The power consumption of light-emitting diodes (LEDs) is low compared with conventional light-emitting materials (incandescent lamps, fluorescent lamps, etc.), and LEDs are long-lasting and have a high emission efficiency (Chang et al., 2012; Liang et al., 2018; Schubert and Kim, 2005; Slocum, 2005; Steranka et al., 2002). Therefore, applications are expanding to automobile headlights, traffic signals, and outdoor displays, which need to be used for a long period (Beng, 2012; Kim et al., 2013; Luo et al., 2016; Okumura, 2011; Yanagisawa and Kojima, 2004). For the long-term use of LEDs, LED sealants are usually applied. LED sealants are used to protect light-emitting elements from external deterioration factors such as heat, moisture, and gas, which lower the LED emission efficiency, and to support a long LED life (Okumura, 2011). The characteristics required for LED sealants include high adhesion to surrounding materials and high light transmittance. In addition, durability against heat and moisture, which deteriorate emission substrates, and low permeation of gas such as water vapor are also important (Okumura and Maeshima, 2010; Okumura, 2011). The material can be classified into two groups: organic (epoxy-based) and inorganic (glass-based) materials. Organic sealants represented by epoxy-based materials (Huang et al., 2004; Li et al., 2013; Okumura and Maeshima, 2010; Schroeder et al., 2013) are excellent in gas-barrier properties. However, they deteriorate (change color) due to light (ultraviolet [UV] light) and heat exposure during continuous use. Thus, there is a drawback in that longterm use is not possible because of the low durability. Inorganic sealants such as glass have high heat resistance and light resistance (Chen et al., 2015; Matsumoto, and Nakamura, 2012; Yoo et al., 2016; Zhang et al., 2014). Thus, they do not deteriorate under a harsh environment such as high-power LED, and long-term use can be expected. However, the softening temperature of glass is relatively high. Thus, there is a possibility that damage to the LED and the deactivation of the element can take place due to the heat load during the sealing process (Matsumoto and Nakamura, 2012). Thus, we focused on the application of organic-inorganic hybrid materials having enhanced characteristics and functions of both materials as LED sealants (Corcione, et al., 2014; Kim, et al., 2010; Schmidt, et al., 2000). Organic-inorganic hybrid materials are materials in which organic and inorganic components are combined at the molecular level. They are promising functional materials that have the merits of both organic and inorganic materials. In this study, we selected a gel of polysilsesquioxane (PSQ) with a silica skeleton, which was prepared from an organic alkoxysilane, as the inorganic component, and an acrylic monomer, which has a carbon double bond, as the organic component. Our PSQ is PSQ-SH, which has a mercapto group (-SH group) at the terminal, and a thiol-ene reaction takes place with a double bond $(\mathrm{C}=\mathrm{C})$ in the monomer upon UV irradiation (wavelength range: 312-570 nm) (Kim, et al., 2013; Liu, et al., 2018; Matsukawa et al., 2010). Therefore, easy curing can be achieved by just irradiating light. As the monomer to be added, ethylene 
glycol dimethacrylate (EGDMA), trimethylolpropane trimethacrylate (TRIM), and ditrimethylolpropane tetraacrylate (DTMPTA), in which the number of contained functional groups is 2, 3, and 4, respectively, were selected. Material curing with light leads to a reduction in heat load on the emission substrate during sealing. In addition, the sealing process is simpler than heat sealing. Therefore, a reduction in production cost can be expected. The objective of this study is to develop LED sealants that can be easily cured by light. The sealing strength, durability, transparency, and gas-barrier properties of the prepared hybrid materials were evaluated to check the adhesion to a surrounding material, durability, and high transparency, which are required for LED sealants. In the present paper, we reported the effect of the number of double bonds in the acrylic monomer on the properties of the hybrid materials.

\section{Experiment}

\section{$2.1 \quad$ Reagents}

3-Mercaptopropyltrimethoxysilane, ethylene glycol dimethacrylate (EGDMA), trimethylolpropane trimethacrylate (TRIM), and ditrimethylolpropane tetraacrylate (DTMPTA) were purchased from Tokyo Kasei Co. (TCI). Polymerization inhibitor was removed from the respective monomers (EGDMA, TRIM, and DTMPTA) with $10 \%$ sodium hydroxide aqueous solution. Then, they were washed with distilled water until the PH became neutral before use.

\subsection{Preparation of PSQ-SH}

The preparation of PSQ-SH is outlined in Figure 1. 3Mercaptopropyltrimethoxysilane, ultra-pure water, and formic acid, as the catalyst, were added to the solvent toluene, and a hydrolysis reaction was carried out by stirring at room temperature for $30 \mathrm{~min}$. Subsequently, dehydration condensation was carried out by stirring at $70^{\circ} \mathrm{C}$ for $2 \mathrm{~h}$; thus PSQ-SH was prepared by removing the impurities, namely toluene, methanol, ultra-pure water, and formic acid with an evaporator under reduced pressure.

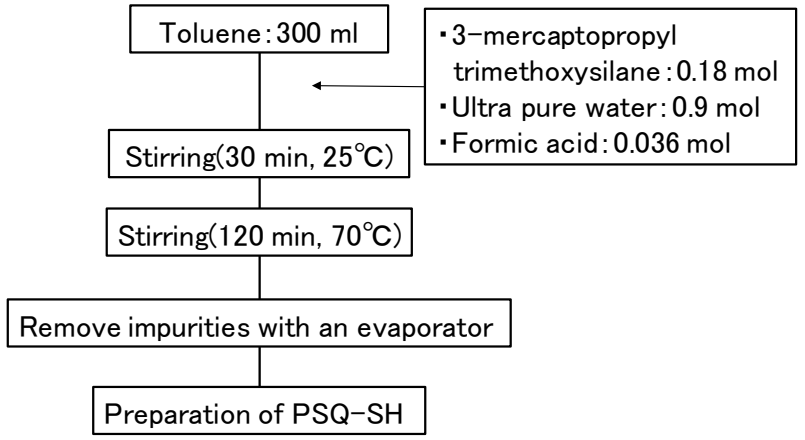

Figure 1. Preparation of PSQ-SH
The preparation for the hybrid materials is outlined in Figure 2. PSQ-SH and EGDMA, TRIM, or DTMPTA were mixed, stirred at room temperature for 30-60 sec, and then shaped into a form suitable for each measurement. Subsequently, they were cured by UV irradiation (wavelength range: $312-570 \mathrm{~nm}$ ) with a UV lamp (UVL-IOOP, Rikoh Kagaku Co., Ltd., output: 100 W). Hardening was observed by scratching UV-irradiated samples with a spatula; it was determined to be cured when a scratch was observed.

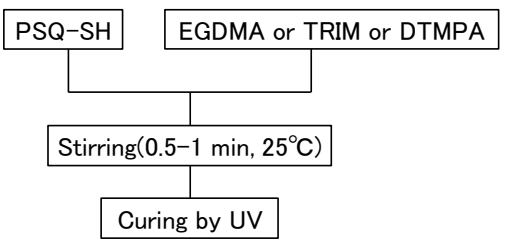

Figure 2. Preparation of hybrid materials

\subsection{Thermogravimetry-differential thermal analyzer (TG-DTA) analysis}

The temperature at which the weight of the hybrid material starts to decrease drastically (onset temperature of weight loss $\left[{ }^{\circ} \mathrm{C}\right]$ ) was determined with a thermogravimetry-differential thermal analyzer TG-DTA (TG-8120, Rigaku Corporation). In the TG measurement, tangent lines were drawn before and after the value drastically decreases, and the intersection point of the tangent lines was judged to be the onset temperature of weight loss $\left[{ }^{\circ} \mathrm{C}\right]$. The measurement range was $25-1000^{\circ} \mathrm{C}$, and $\alpha$-alumina was used as the reference.

\subsection{Sealing strength}

The prepared hybrid material was dropped on soda-lime glass substrate $(40 \times 40 \times 1 \mathrm{~mm})$. Subsequently, another soda-lime glass substrate was superimposed, and the hybrid material was cured by UV irradiation (wavelength range: $312-570 \mathrm{~nm}$ ); thus, the two soda-lime glass substrates were bonded. The sample after sealing was fixed on a Tensilon universal testing machine (RTC1210A, A\&D Company, Limited), and a tensile test was carried out at a rate of $0.3 \mathrm{~mm} / \mathrm{min}$. The obtained measurement value was converted to the sealing strength per $1 \mathrm{~cm}^{2}\left[\mathrm{kgf} / \mathrm{cm}^{2}\right]$.

\subsection{Light transmittance measurement}

A thin film was formed on the soda-lime glass substrate so that the film thickness is $500 \mu \mathrm{m}$. The transmittance $\mathrm{T}$ [\%] of the prepared sample was measured with a UV-VIS spectrophotometer (UV 1700, Shimadzu Corporation). The measurement was carried out in the range of 300-800 nm. Air was used as the reference. 


\subsection{Moisture permeability measurement}

The moisture permeability was measured according to the Japanese Industrial Standards (JIS Z 0208:1976). Hybrid material was applied on a polypropylene film $(200 \mu \mathrm{m})$ so that the thickness is $30 \mu \mathrm{m}$. The moisture permeability of the prepared sample was measured with a $30 \mathrm{ml}$ permeability cup, $6 \mathrm{~g}$ of calcium chloride as the desiccant, and the substrate was polypropylene film. They were allowed to stand in a desiccator at $25^{\circ} \mathrm{C}$ and a humidity of $90 \pm 2 \%$; the weight was measured every $24 \mathrm{~h}$, and the mass $[\mathrm{g}]$ of permeating water vapor was determined per $24 \mathrm{~h}$ and per $1 \mathrm{~m}^{2}$ of the substrate.

\subsection{Light emission test}

In order to study whether the prepared hybrid material can withstand continuous lighting, a light emission test was carried out. As shown in Figure 3, the surface of LED substrate (chip on board type (COB), provided by YEJ glass Co., Ltd.) was sealed with each hybrid material. Subsequently, it was checked if cracks were generated by continuous lighting on the applied hybrid material. The observation was carried out every $12 \mathrm{~h}$, and the measurement was made with a voltage of $30 \mathrm{~V}$ and current of $0.05 \mathrm{~A}$.

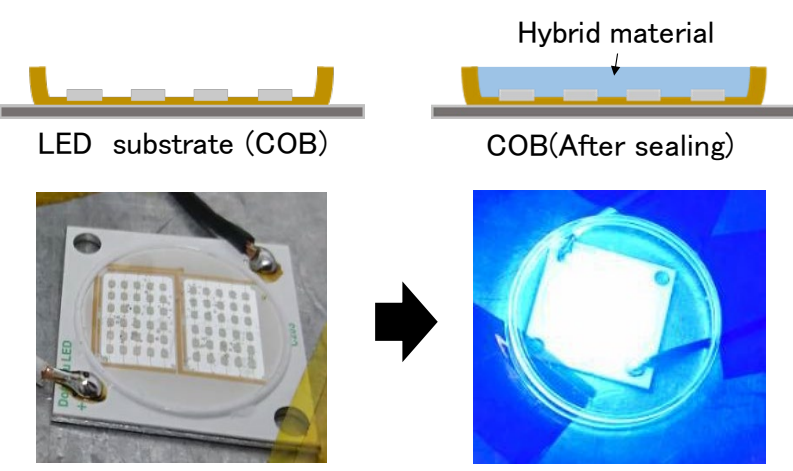

Figure 3. Photographs of LED substrate sealed by hybrid material and light emission test

\section{Results and Discussion}

\subsection{Thermogravimetry-differential thermal analyzer (TG-DTA) analysis}

Curing of the prepared PSQ-SH/EGDMA hybrid material (PEH), PSQ-SH/TRIM hybrid material (PTH), and PSQSH/DTMPTA hybrid material (PDH) was completed in about $5 \mathrm{~min}$, respectively. This means that even a low heat load during light irradiation can be drastically reduced in the sealing process of light-emitting elements with low heat resistance. In order to observe the durability of each hybrid material, TG-DTA measurement was carried out. As seen from the results in Fig. 4, the rate of weight loss [\%] was the best for PDH. This may be because that the polymerization density of the hybrid material became high by using a monomer with four functional groups. However, a large difference in the onset temperature of weight loss was not observed. The results indicate that factors that reduce thermal properties of our hybrid materials are due to monomers and unreacted functional groups of PSQ-SH and the hydroxy group (-OH group) at the PSQ-SH terminal (Shang et al., 2018).

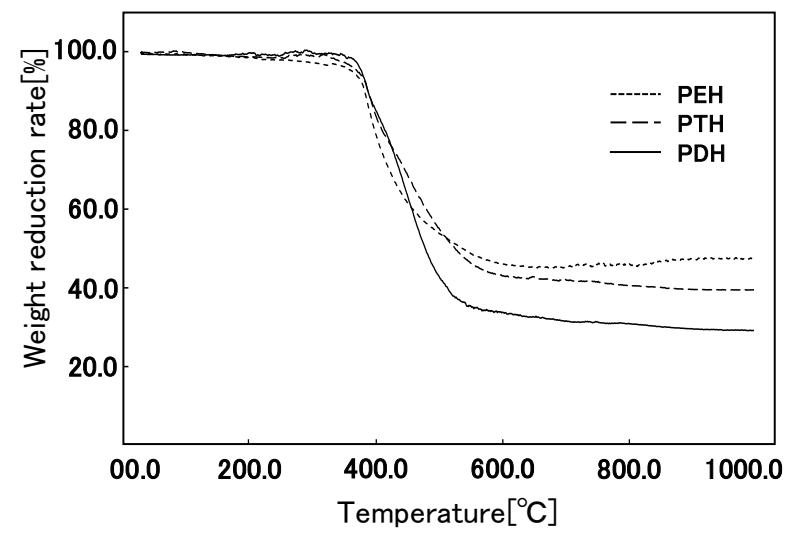

Figure 4. Effect of added monomer to PSQ-SH on heat resistance

\subsection{Sealing strength measurement}

Adhesion of hybrid materials to surrounding materials was investigated by bonding two soda-lime glass substrates and observing the adhesion (Figure 5). According to the results, the mechanical strength of the hybrid material greatly improved with an increase in the number of monomer functional groups. In particular, the sealing strength exceeded $15\left[\mathrm{kgf} / \mathrm{cm}^{2}\right]$ with PDH for which DTMPTA was used as the monomer. A commercial lead glass, which is a general sealant of electro devices, is about $3 \mathrm{kgf} / \mathrm{cm}^{2}$ in the sealing strength (Yoshida et al., 2004). As the prepared hybrid material is about $15 \mathrm{kgf} / \mathrm{cm}^{2}$ in the sealing strength, the material has enough sealing strength compared to commercial sealing materials. Sealing consists of an anchor effect, intermolecular interactions, and chemical bonds (Minamizaki, 2003). A chemical bond forms a hydrogen bond between the $-\mathrm{OH}$ group on the glass surface and the $-\mathrm{OH}$ group of PSQ-SH. The -OH groups on the glass surface can also form hydrogen bonds with the acrylate groups of the hydrogen bond acceptor. For this reason, DTMPTA, which forms multiple hydrogen bonds, is considered to have a larger adhesion interface area with glass than TRIM. Moreover, since the curing of the material by the thiol-ene reaction can reduce the curing shrinkage, it is considered that the decrease in the sealing strength when the polyfunctional monomer is used is suppressed. In addition, the fluidity of hybrid material is high before curing. Therefore, when soda-lime glass and soda-lime glass are bonded, the anchoring is effective at increasing the sealing strength. This may also be the reason for the high sealing strength of hybrid material. Thus, the hybrid materials in this study are compatible 
with glass substrates. Accordingly, other applications may be also possible as well as LED sealants.

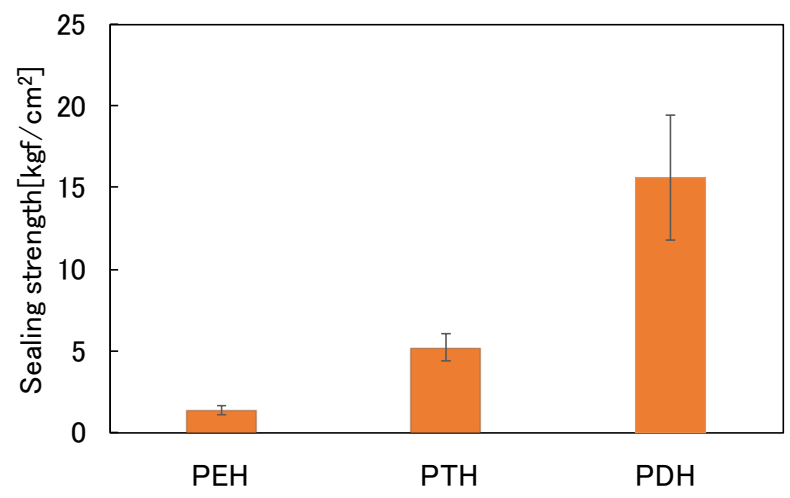

Figure 5. Effect of monomer added to PSQ-SH on sealing strength

\subsection{Light transmittance measurement}

In order to utilize the high emission efficiency of LEDs, light transparency is an important property required for LED sealants. The results for light transmittance measurements for each hybrid material are shown in Fig. 6. According to the results, comparable light transmittance to that of the substrate soda-lime glass could be obtained for PTH and PDH. Thus, the strength could be improved, while maintaining transparency, by using DTMPTA as the monomer. Based on the results, when this hybrid material is used as the LED sealant, the emission substrate can be sealed without a loss of light from the light-emitting elements.

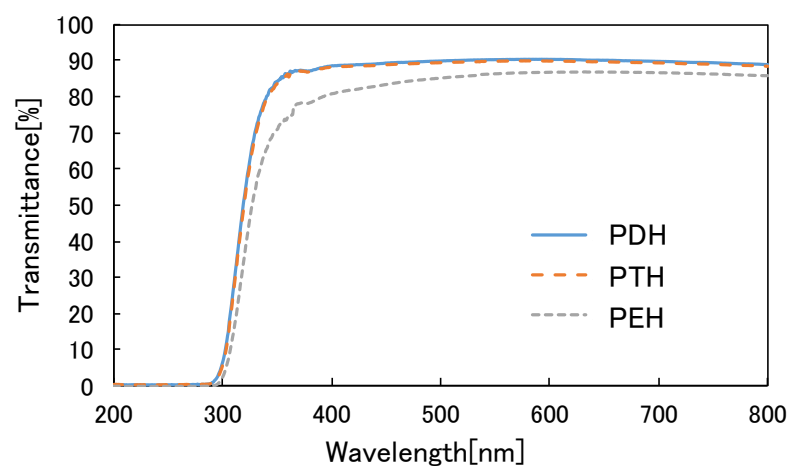

Figure 6. Light transmittance measurement

\subsection{Moisture permeability measurement}

Regarding the characteristics required for LED sealants, the protection of substrate elements from water vapor, oxygen, and sulfur gas is important. Thus, the water molecule, which has the smallest molecular size among these gases, was focused on, and its moisture permeability was measured. If the permeation of water molecules (water vapor) can be suppressed, the permeation of other gas molecules can also be physically suppressed. Because a measurement with only the hybrid material could not be carried out, polypropylene film was used as the substrate. According to the measurement results for moisture permeability (Figure 7), the permeation of water vapor was suppressed by the hybrid materials, even though they were thinner than the polypropylene film substrate. In addition, there was no large difference due to the number of functional groups of the monomer in the suppression of water vapor permeation. Although the hybrid materials in this study suppressed gas permeation, the moisture permeability was still large when we consider that the moisture permeability of silica-based thin film is $10^{-1}$ $\left[\mathrm{g} / \mathrm{m}^{2} \cdot\right.$ day] (Hara, 2013). This may be because the permeation of gas depends on the pore size of the covering film (Stolyarchuk, 2018). The siloxane skeleton of our PSQ-SH takes an amorphous structure, and the network structure is larger than a water molecule (Kanezashi, 2011; Kinoshita et al., 2010). As a result, a drastic suppression of the permeation of water vapor could not be achieved. This property may be improved by introducing bulky aromatic functional groups into PSQ-SH.

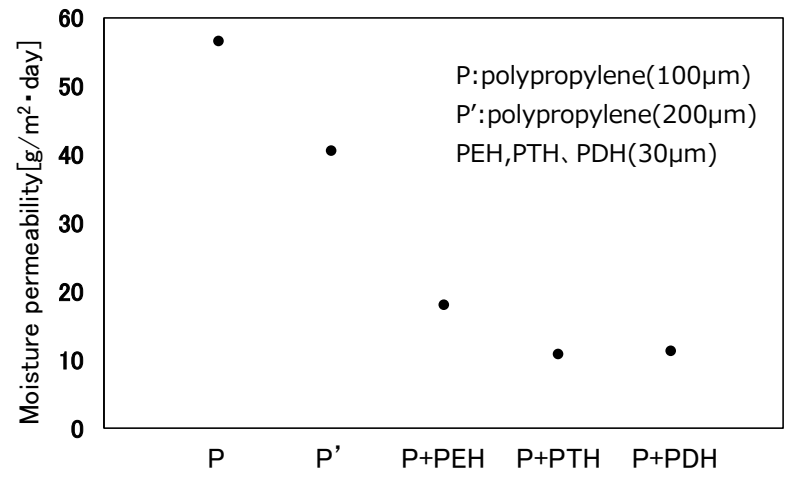

Figure 7. Moisture permeability measurement

\section{5 light emission test}

A light emission test was carried out to observe whether cracks are generated during lighting when the prepared hybrid material is applied as the LED sealant (Figure 8). The results are shown in Table 1. As seen in the table, the generation of cracks occurred to a lesser degree when PDH was used as the sealant compared with when PEH or PTH was used. In the case of PEH, lighting was stopped after the generation of cracks; the material was found to have turned yellow. This may be due to its low heat resistance compared with other hybrid materials, as seen in the TG-DTA measurement results. However, when lighting was stopped before the generation of cracks in lighting, cracks were later generated in all the hybrid materials. This is considered to be caused by a large stress generated by the thermal expansion of the hybrid material, due to heat (about $90^{\circ} \mathrm{C}$ ) from the emission substrate, and subsequent cooling after turning the light off. This property may be improved by adding filler that has a negative thermal expansion coefficient (Hsueh et al., 2003). 
Table 1. Results of light emission test

\begin{tabular}{|c|c|c|c|}
\hline \multirow{2}{*}{} & \multicolumn{3}{|c|}{ Lighting time[hours] } \\
\cline { 2 - 4 } & $12 \mathrm{~h}$ & $24 \mathrm{~h}$ & $36 \mathrm{~h}$ \\
\hline PEH & $\bigcirc$ & $\bigcirc$ & $\times$ \\
\hline PTH & $\bigcirc$ & $\bigcirc$ & $\times$ \\
\hline PDH & $\bigcirc$ & $\bigcirc$ & $\bigcirc$ \\
\hline
\end{tabular}
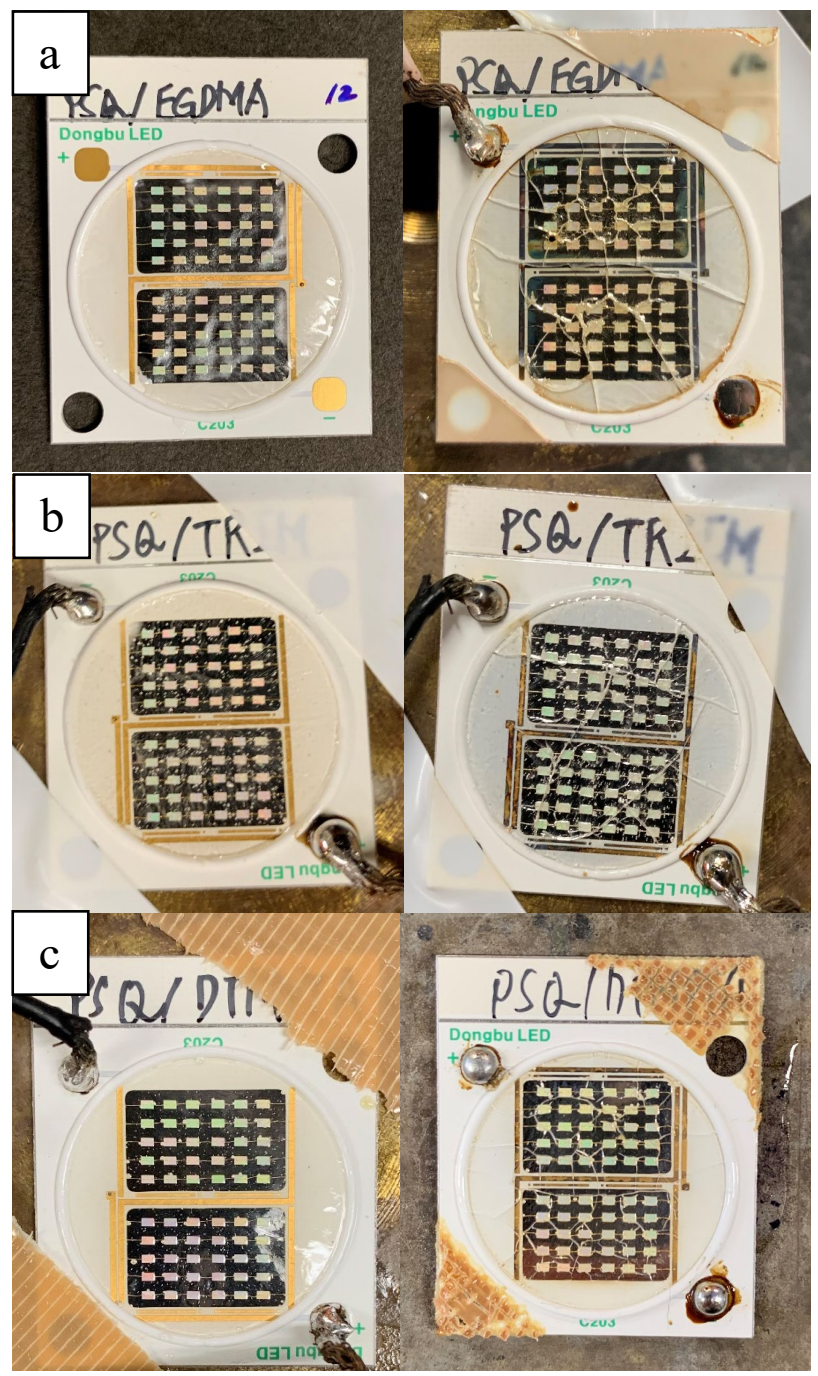

Figure 8. Results of light emission test (left: before lighting, right: after lighting): (a) PEH, (b) PTH, (c) PDH

\section{Conclusion}

Curing was possible for all the hybrid materials (PEH, $\mathrm{PTH}$, and PDH) with UV irradiation of only about $5 \mathrm{~min}$. The heat resistance and mechanical strength were improved by increasing the number of monomer functional groups. PTH and PDH exhibited high transparency comparable to the soda-lime glass the substrate. The heat resistance and mechanical strength were improved with the use of PDH while maintaining the transparency level of PTH. According to the moisture permeability experiments, the permeation of water vapor could be suppressed by the hybrid materials compared with a plastic material used as the substrate. However, the permeation of water vapor was not sufficiently suppressed for the application to LED sealants. In the light emission test, the generation of cracks during lighting was suppressed more with PDH than with PTH. However, cracks were generated after a long lighting duration. Cracks were also generated by the stress caused by the contraction, when the light was turned off, because they had high thermal expansion coefficients. The hybrid materials in this study have high sealing capability and light transparency; therefore, they are promising as LED sealants if the suppression of water vapor permeation can be improved and the generation of cracks during lighting can be suppressed.

\section{References}

Beng, G.; "UV-curable Encapsulants for LED," Orient. $J$. Chem., 28, 1135-1140 (2012)

Chang, M.-H., D. Das, P.V. Varde, and M. Pecht; "Light Emitting Diodes Reliability Review," Microelectronics Reliability, 52 ,762-782 (2012)

Chen, D., W. Xiang, X. Liang, J. Zhong, H. Yu, M. Ding, H. Lu, and Z. Ji; "Advances in Transparent GlassCeramic Phosphors for White Light-Emitting Diodes-A Review," Journal of the European Ceramic Society, 35, 859-869 (2015)

Corcione, C. E., R. Striani, and M. Frigione; "OrganicInorganic UV-cured Methacrylic-Based Hybrids as Protective Coatings for Different Substrates," Progress in Organic Coatings, 77, 1117-1125 (2014)

Hara, D.; "Development of Gas Barrier Materials," Tosoh research \& technology review, 57, 39-44 (2013)

Hsueh, H.-B., C.-Y. Chen, C.-C. Wang, and T.-J. Chu; "Preparation and Properties of APPSSQ-like/Polyimide Hybrid Composites," Journal of Applied Polymer Science, 89, 2865-2874 (2003)

Huang, J.-C., Y.-P. Chu, M. Wei, and R. D. Deanin; "Comparison of Epoxy Resins for Applications in LightEmitting Diodes," Adv. in Polym. Technol., 23, 298-306 (2004)

JIS Z 0208:1976 Testing Methods for Determination of the Water Vapour Transmission. Rate of Moisture -Proof Packaging Materials (Dish Method), JIS (1976)

Kanezashi, M.; "Sol-Gel Derived Silica Membranes: Control of Silica Network Size and Hydrothermal Stability," MEMBRANE, 36, 97-103 (2011)

Kim, J., B. Ma, and K. Lee; "Comparison of Effect of Epoxy and Silicone Adhesive on the Lifetime of Plastic LED Package," Electronic Materials Letters, 9, 429-432 (2013) 
Kim, J.-S., S. C. Yang, and B.-S. Bae; "Thermally Stable Transparent Sol-Gel Based Siloxane Hybrid Materialwith High Refractive Index for Light Emitting Diode (LED) Encapsulation," Chem. Mater., 22, 3549-3555 (2010)

Kim, S. K., C. Baguenard, and C. A. Guymon; "Influence of Photopolymerization Characteristics on ThermoMechanical Properties of Nanocomposites Utilizing Polymerizable Organoclays in Thiol-Acrylate Systems," Macromol. Symp., 329, 173-192 (2013)

Kinoshita, Y., H. Ashihara, A. Hashimoto, and K. Kuraoka; "Preparation of Silica/Poly (Vinyl Alcohol) Hybrid Gas Barrier Membrane with Cross-Linked Structure-Effect of Addition of Maleic Acid," Journal of Packaging Science \& Technology, Japan, 20, 35-41 (2010)

Li, T., J. Zhang, H. Wang, Z. Hu, and Y. Yu; "HighPerformance Light-Emitting Diodes Encapsulated with Silica-Filled Epoxy Materials," Appl. Mater. Interfaces, 5, 8968-8981 (2013)

Liang, L., C. Zeng, X. Xiaohui, C. Guolong, Y. Rogqian, Z. Rui, Z. Lei, M. Yu, and Y. Min; "Effects of Oxidation Curing and Sintering Temperature on the Microstructure Formation and Heat Transfer Performance of Freestanding Polymer-Derived SiC Films for High-Power LEDs," Ceramics International, 44, 6072-6080 (2018)

Liu, H., Z. Wang, and C. Sun; "Robust Water-Repellent Treatment of Cotton Fabrics with Polysiloxane Modified via Thiol-Ene Click Reaction," Fibers and Polymers, 19, 580-586 (2018)

Luo, X. B., R. Hu, S. Liu, and K. Wang; "Heat and Fluid Flow in High-Power LED Packaging and Applications," Prog. Energy Combust. Sci., 56, 1-32 (2016)

Matsukawa, K., T. Fukuda, S. Watase, and H. Goda; "Preparation of Photo-curable Thiolene Hybrids and their Application for Optical Materials," Journal of Photopolymer Science and Technology, 23, 115-119 (2010)

Matsumoto, S., and N. Nakamura; "Glass for LED encapsulation," NEW GLASS, 27, 11-14 (2012)

Minamizaki, Y.; "Adhesives and Adhesion Mechanism," Journal of Japan Institute of Electronics Packaging, 6, 349-354 (2003)

Okumura, K. and H. Maeshima; "Recent Improvement in the Epoxy Resins for LED Encapsulant," Journal of the Adhesion Society of Japan, 46, 401-405 (2010)

Okumura, K.; "The Latest Developmental Trend of LED Encapsulants," Journal of Japan Institute of Electronics Packaging, 14,175-178 (2011)

Schmidt, H., G. Jonschker, S. Goedicke, and M. Mennig; "The Sol-Gel Process as a Basic Technology for Nanoparticle-Dispersed Inorganic-Organic Composites,"
Journal of Sol-Gel Science and Technology, 19, 39-51 (2000)

Schroeder, W. F., I. E. dell'Erba, G. F. Arenas, S. V. Asmussen and C. I. Vallo; "Encapsulants for LightEmitting Diodes from Visible Light-Cured Epoxy Monomers," Polym. Adv. Technol., 24,430-436 (2013)

Schubert, E. F. and J. K. Kim; "Solid-State Light Sources Getting Smart," Science, 308, 1274-1278 (2005)

Shang, X. X., S. Duan, M. Zhang, X. Y. Cao, K. Zheng, J. N. Zhang, Y. M. Ma, and R. B. Zhang; "UV-Curable Ladder-Like Diphenylsiloxane-Bridged MethacrylPhenyl-Siloxane for High Power LED Encapsulation," RSC Adv., 8, 9049-9056 (2018)

Slocum, A.; A Technology Assessment of Light Emitting Diode (LED) Solid State Lighting for General Illumination, National Center for Environmental Economics (NCEE), Washington, DC (2005)

Steranka, F. M., J. Bhat, D. Collins, L. Cook, M. G. Craford, R. Fletcher, N. Gardner, P. Grillot, W. Goetz, M. Keuper, R. Khare, A. Kim, M. Krames, G. Harbers, M. Ludowise, P. S. Martin, M. Misra, G. Mueller, R. Mueller-Mach, S. Rudaz, Y. C. Shen, D. Steigerwald, S. Stockman, S. Subramanya, T. Trotter, and J. J. Wierer; "High Power LEDs-Technology Status and Market Applications," Phys. Status Solidi a-Appl.Mater. Sci., 194, 380-388 (2002)

Stolyarchuk, N. V., H. Kolev, M. Kanuchova, R. Keller, M. Vaclavikov, and I. V. Melnyk; "Synthesis and Sorption Properties of Bridged Polysilsesquioxane Microparticles Containing 3-Mercaptopropyl Groups in the Surface Layer," Colloids and Surfaces A, 538, 694702 (2018)

Yanagisawa, T. and T. Kojima; "Long-Term Accelerated Current Operation of White Light-Emitting Diodes," Journal of Luminescence, 114, 39-42 (2005)

Yoo, H., Y. Kouhara, H. C. Yoon, S. J. Park, J. H. Oh, and Y. R. Do; "Sn-P-F Containing Glass Matrix for the Fabrication of Phosphor-in-Glass for Use in High Power LEDs," RSC Adv., 6, 111640-111647 (2016)

Yoshida, M., T. Honda, Y. Uemura, Hatate, Y., and T. Sarata; "Formation of Lead-Free Sealing Glasses in the Quaternary System $\mathrm{V}_{2} \mathrm{O}_{5}-\mathrm{ZnO}-\mathrm{BaO}-\mathrm{TeO}_{2}$, , Kagaku Kogaku Ronbunshu, 30, 233-239 (2004)

Zhang, R., H. Lin, Y. Yu, D. Chen, J. Xu, and Y. Wang; "A New-Generation Color Converter for High-Power White LED: Transparent Ce3+: YAG Phosphor-in-Glass," Laser and Photonics Reviews, 8, 158-164 (2014) 